\title{
Prognostic value of chest computed tomography in community-acquired pneumonia patients
}

\author{
Masahiro Nemoto ${ }^{1,2}$, Kei Nakashima1, Satoshi Noma ${ }^{3}$, Yuya Matsue ${ }^{4}$, \\ Kazuki Yoshida ${ }^{5}$, Hiroki Matsui ${ }^{6}$, Atsushi Shiraishi ${ }^{7}$, Tomoko Ishifuji ${ }^{8}$, \\ Konosuke Morimoto ${ }^{8}$, Koya Ariyoshi $\mathbb{1}^{8}$ and Masahiro Aoshima ${ }^{1}$
}

Affiliations: ${ }^{1}$ Dept of Pulmonary Medicine, Kameda Medical Center, Kamogawa, Japan. ${ }^{2}$ Dept of Immunology, Graduate School of Medicine, Chiba University, Chiba, Japan. ${ }^{3}$ Dept of Pulmonary Medicine, Shinkuki General Hospital, Kuki, Japan. ${ }^{4}$ Dept of Cardiovascular Medicine, Juntendo University, Tokyo, Japan. ${ }^{5}$ Division of Rheumatology, Immunology and Allergy, Dept of Medicine, Brigham and Women's Hospital and Harvard Medical School, Boston, MA, USA. ${ }^{6}$ Clinical Research Support Division, Kameda Institute for Health Science, Kameda College of Health Sciences, Kamogawa City, Japan. ${ }^{7}$ Emergency and Trauma Center, Kameda Medical Center, Kamogawa, Japan. ${ }^{8}$ Dept of Clinical Medicine, Institute of Tropical Medicine, Nagasaki University, Nagasaki, Japan.

Correspondence: Masahiro Nemoto, Dept of Pulmonary Medicine, Kameda Medical Center, 929 Higashi-cho, Kamogawa, Japan. E-mail: mshr.nmt.13đggmail.com

\section{ABSTRACT}

Background: Chest computed tomography (CT) is commonly used to diagnose pneumonia in Japan, but its usability in terms of prognostic predictability is not obvious. We modified CURB-65 (confusion, urea $>7 \mathrm{mmol} \cdot \mathrm{L}^{-1}$, respiratory rate $\geqslant 30$ breaths $\cdot \mathrm{min}^{-1}$, blood pressure $<90 \mathrm{mmHg}$ (systolic) $\leqslant 60 \mathrm{mmHg}$ (diastolic), age $\geqslant 65$ years) and A-DROP scores with CT information and evaluated their ability to predict mortality in community-acquired pneumonia patients.

Methods: This study was conducted using a prospective registry of the Adult Pneumonia Study Group Japan. Of the 791 registry patients, 265 hospitalised patients with chest CT were evaluated. Chest CTmodified CURB-65 scores were developed with the first 30 study patients. The 30-day mortality predictability of CT-modified, chest radiography-modified and original CURB-65 scores were validated.

Results: In score development, infiltrates over four lobes and pleural effusion on CT added extra points to CURB-65 scores. The area under the curve for CT-modified CURB-65 scores was significantly higher than that of chest radiography-modified or original CURB-65 scores (both $\mathrm{p}<0.001$ ). The optimal cut-off CTmodified CURB-65 score was $\geqslant 4$ (positive-predictive value $80.8 \%$; negative-predictive value $78.6 \%$, for 30 day mortality). For sensitivity analyses, chest CT-modified A-DROP scores also demonstrated better prognostic value than did chest radiography-modified and original A-DROP scores. Poor physical status, chronic heart failure and multiple infiltration hampered chest radiography evaluation.

Conclusion: Chest CT modification of CURB-65 or A-DROP scores improved the prognostic predictability relative to the unmodified scores. In particular, in patients with poor physical status or chronic heart failure, CT findings have a significant advantage. Therefore, CT can be used to enhance prognosis prediction.

@ERSpublications

Chest CT modification of CURB-65 and A-DROP improves prognosis prediction in communityacquired pneumonia. Patients with low physical status or chronic heart failure may have mismatch of chest CT and radiography findings. https://bit.ly/30GbNZS

Cite this article as: Nemoto M, Nakashima K, Noma S, et al. Prognostic value of chest computed tomography in community-acquired pneumonia patients. ERJ Open Res 2020; 6: 00079-2020 [https:// doi.org/10.1183/23120541.00079-2020].

This article has supplementary material available from openres.ersjournals.com

Received: 19 Feb 2020 | Accepted after revision: 19 July 2020

Copyright $\odot$ ERS 2020. This article is open access and distributed under the terms of the Creative Commons Attribution Non-Commercial Licence 4.0. 
Community-acquired pneumonia (CAP) is one of the four major causes of death worldwide [1]. Pneumonia-related mortality in older individuals ( $\geqslant 70$ years) increased by $33.6 \%$ in the decade leading up to 2017 [1], and so prognostic prediction is crucial for determining the site of care of patients with pneumonia and for avoiding unnecessary hospital admission [2]. The CURB-65 (confusion, urea $>7 \mathrm{mmol} \cdot \mathrm{L}^{-1}$, respiratory rate $\geqslant 30$ breaths $\cdot \mathrm{min}^{-1}$, blood pressure $<90 \mathrm{mmHg}$ (systolic) $\leqslant 60 \mathrm{mmHg}$ (diastolic), age $\geqslant 65$ years), which assigns scores for confusion, uraemia, respiratory rate, blood pressure and age $\geqslant 65$ years [3], is the most widely known scoring system and, given its simplicity, European guidelines recommend its use after a patient is diagnosed with CAP in a hospital care setting [3, 4].

Another useful prognostic scoring system, the A-DROP, proposed by the Japanese Respiratory Society, uses scores that are similar to those of the CURB-65, but with a different cut-off age: $\geqslant 70$ years for men and $\geqslant 75$ years for women [5]. Some studies in Japan and the US have reported a slightly higher area under the receiver operating characteristic curve (AUROC) for the A-DROP scoring system as compared to the CURB-65 $[6,7]$.

A score of $\geqslant 2$ on the CURB-65 generally indicates the need for admission and has high sensitivity (92.8\%) for 30-day mortality, but its specificity $(49.2 \%)$ is unsatisfactory [8]. Thus, in practice, clinicians always require supplemental information to improve the specificity for prognosis and to determine whether CAP patients should be hospitalised or can be treated as outpatients. Indeed, the updated US guidelines suggest further investigation of the CURB-65 scores and new prediction rules for prognosis [9].

Chest computed tomography (CT) has been reported as a useful diagnostic tool for detecting pneumonia when chest radiography could not detect abnormal infiltration in the lung field [10]. Additionally, chest $\mathrm{CT}$ is helpful in detecting complications in pneumonia or the specific aetiology of pneumonia [11]. Careful differentiation of pneumonia from non-infectious diseases, including heart failure, interstitial lung disease and chronic obstructive pulmonary disease, is also difficult with chest radiograph alone [12]

In terms of prognostic assessment, some prospective studies have reported that chest radiograph findings are useful for predicting the prognosis of CAP [13-15]. In fact, these findings have been included in some of the major prognostic scoring systems, including the Pneumonia Severity Index (PSI) [2] and SMART-COP [10]. In contrast, there are currently no data available to verify the prognostic predictability of chest CT in pneumonia cases [2]. The quantity of infiltrates and ancillary findings on chest CT is easily considered for the prognosis of pneumonia; however, this was merely an extrapolation of evidence from chest radiography [13-15]. Therefore, the objective of this study was to evaluate the ability of CURB-65 scores modified with CT information to predict mortality in CAP patients.

\section{Methods}

\section{Study design and study participants}

This study was a secondary analysis of the Adult Pneumonia Study Group - Japan registry, a multicentre prospective surveillance programme conducted from 2011 to 2013 at four hospitals located in the major islands of Japan $[16,17]$.

The current study targeted patients registered in the cohort at Kameda Medical Center, Chiba, Japan, a 925-bed tertiary care centre that is the largest of the four participating facilities, from February 2012 to January 2013. Patients were enrolled who met the following criteria: 1) age $\geqslant 15$ years; 2) symptoms compatible with pneumonia (e.g. fever, cough, sputum, pleuritic chest pain and dyspnoea); 3) presence of new pulmonary infiltrates on chest radiograph or chest CT that were consistent with pneumonia [16]; 4) chest CT performed at the emergency or outpatient department within $48 \mathrm{~h}$ of hospital visit; and 5) hospitalisation. Exclusion criteria were: 1) hospitalisation for reasons other than pneumonia; 2) patients with advanced bronchiectasis or interstitial pneumonia on chest CT classified as severe because the observer could not assess the images; and 3) patients with missing values for calculation of CURB-65 scores.

The Kameda Medical Center institutional review board approved the study (ID 14-042) and allowed the opt-out method instead of requiring informed patient consent.

\section{Chest radiography and chest CT acquisition}

Chest radiography was performed using a standardised protocol with specified position (standing or sitting) and we evaluated the frontal view only, because the lateral view is rarely taken in elderly patients who had difficulty walking. A multidetector chest CT scan was performed using a standard-dose protocol with 2 - to $5-\mathrm{mm}$ collimation at the same $\mathrm{mm}$ intervals. Contrast material was not used in all cases. We acquired prior chest radiograph and CT results if possible, to evaluate whether the lung infiltrates or pleural effusion were new. Because our facility was the only hospital for tertiary care in our city, study participants without prior chest imaging were very rare. 
Index test and reference standard

Patients' chest radiograph and CT images were evaluated and scored independently by two senior chest physicians (K.N. and S.N.) who were blinded to clinical information. Consensus was reached by discussion in the case of disagreement. The observers assessed multilobar chest radiograph involvement, consolidation affecting more than two-thirds of the unilateral lung field or affecting bilateral lung fields, and the presence of pleural effusion, based on previous studies [13-15]. The presence of each of these contributed an additional point to the CURB-65 scores (i.e. the chest radiography-modified CURB-65 scores).

We defined the study reference standard as 30-day mortality from the day of admission.

\section{Score development and validation}

For chest CT analysis, the number of lobes involved and the presence of pleural effusion were assessed in a way similar to a previous study using chest radiograph imaging [13-15]. One observer (K.N.) reviewed the chest CT and the outcome of the first 30 consecutive patients who registered as study participants to establish the scoring system. When determining features that could be used to modify the CURB-65, we considered those that would be clinically amenable. Each of these features was assigned one whole additional point, rather than using statistical weighting, to facilitate subsequent analysis [8]. The scores were then used for validation in the remaining study participants.

\section{Statistical analysis}

The characteristics of both the study and the overall registry participants from Kameda Medical Center were described to shed light on potential selection bias. Categorical variables, expressed as the total number and percent, were compared using chi-squared tests. Continuous variables, expressed as the median and interquartile range, were compared using the Mann-Whitney U-test. Kappa coefficients were used to estimate inter-rater agreement. AUROC was calculated to compare the ability of chest CT-modified CURB-65 scores to discriminate CAP with that of chest radiography-modified and original scores. DeLong's test was used to compare ROC curves. The confidence intervals for the different AUROCs were calculated by bootstrapping (100000 iterations). To control the family-wise error rate of multiple comparisons to $5 \%$, we used a Bonferroni-corrected threshold of $\mathrm{p}<0.017$. As a sensitivity analysis, the A-DROP score was also tested using the same scoring method as that for the CURB-65. All analyses were performed using R ver. 3.5.2 (R Foundation for Statistical Computing, Vienna, Austria) [18] with the add-on package EZR ver. 1.36 (Saitama Medical Center, Jichi Medical University, Saitama, Japan) [19].

\section{Results}

\section{Study participants}

In total, 791 participants were enrolled in the Adult Pneumonia Study Group - Japan registry at Kameda Medical Center, from February 2012 to January 2013; of these, 265 patients were included in the current study. The first 30 of the 265 patients were selected for score development and the remaining 235 patients were used for validation (figure 1). The baseline characteristics of the 265 eligible patients and overall registry population are summarised in table 1. Old age, male sex, low Eastern Cooperative Oncology Group (ECOG) performance status, presence of certain comorbidities and CURB-65 scores were statistically more frequent in the eligible patients than in the total registry population.

\section{Chest CT score development and validation}

In score development using the first 30 patients, the expert panel (K.N. and M.A.) decided to add a single point to the original CURB-65 or A-DROP score for identification of infiltration affecting $\geqslant 4$ lobes and the presence of pleural effusion (unilateral or bilateral) on chest CT, mainly because of correlation with the 30 -day mortality and because infiltration affecting $\geqslant 4$ lobes automatically corresponded to chest radiograph involvement affecting the bilateral lungs.

Subsequently, the 30-day mortality prediction was tested by chest CT- and chest radiography-modified scores in 235 participants (table 2). For both infiltration and pleural effusion, a CT-modified score of 1 significantly correlated with a higher mortality rate, as compared with a score of 0 ; however, for both systems, a chest radiography-modified score of 1 did not correlate with the mortality rate, as compared with a score of 0 .

The inter-rater agreement for chest CT assessment was good for both the presence of unilateral effusion $(\kappa=0.88)$ and involvement of $\geqslant 4$ lobes $(\kappa=0.71)$. For chest radiography observation, the agreement was moderate for multilobar involvement $(\kappa=0.46)$ and fair for the presence of unilateral pleural effusion $(\kappa=0.35)$. 
FIGURE 1 Patient selection flow chart. CT: computed tomography; CURB-65: confusion, urea $>7 \mathrm{mmol} \cdot \mathrm{L}^{-1}$, respiratory rate $\geqslant 30$ breaths $\cdot \mathrm{min}^{-1}$, blood pressure $<90 \mathrm{mmHg}$ (systolic) $\leqslant 60 \mathrm{mmHg}$ (diastolic), age $\geqslant 65$ years
February 2012 - January 2013

791 cases of community-acquired pneumonia

338 patients; outpatient clinic

264 patients; without chest CT

340 hospitalised patients with chest CT

59 patients; primary reason for hospitalisation was not pneumonia

22 patients; patients with advanced bronchiectasis, or interstitial pneumonia

26 patients; lack of data for CURB-65 calculation

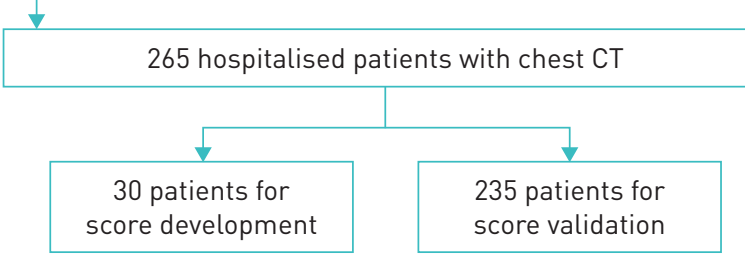

(table 3); there were no significant differences between that of the chest radiography-modified CURB-65 or A-DROP scores and that of the original CURB-65 or A-DROP scores, respectively. The AUROC of PSI also showed no statistically significant difference as compared to that of the original CURB-65 scores (figure S1).

The sensitivity, specificity and positive- and negative-predictive values for 30 -day mortality for all scoring models are shown in table S1. A CURB-65 score of 2, which is the recognised cut-off value for hospitalisation in general [2], has $82.8 \%$ sensitivity with specificity of only $38.8 \%$; in contrast, a chest CT-modified A-DROP score of 4 (sensitivity=79.3\%) showed the highest specificity $(89.8 \%)$. Regarding predictive value, chest CT-modified CURB-65 and A-DROP scores of 0-3 showed remarkable negative-predictive value $(>94 \%)$ and scores of 4-7 showed good positive-predictive value $(>80 \%)$. In contrast, the original scores showed similar negative-predictive value for scores of $0-2$, but even scores of $4-5$ showed a negative-predictive value of $<75 \%$, which was unsatisfactory.

\section{Discrepancy between chest images and patient characteristics}

There were discrepant findings in the presence of pleural effusion between chest CT and chest radiographs: $19.4 \%$ versus $2.4 \%$ in survivors, and $82.8 \%$ versus $6.9 \%$ in non-survivors. We compared the characteristics of the patients with and without such radiological discrepancy in pleural effusion (table S2). Low ECOG performance status, heart failure as a chronic underlying disease, and chest CT-demonstrated infiltration affecting $\geqslant 4$ lobes were significantly more frequent in patients with this radiological discrepancy.

\section{Discussion}

Prognostic prediction in cases with CAP is crucial for deciding site of care and avoiding unnecessary hospitalisation in the current rapidly aging society. As the prognostic value of chest CT findings has previously been unappreciated and sometimes the evidence was extrapolated from that of chest radiographs, we investigated the prognostic predictability of chest CT information. Chest CT-modified CURB-65 scores had a significantly higher AUROC than did the original and chest radiography-modified CURB-65 scores for predicting 30-day mortality. Moreover, the results of a sensitivity analysis using chest CT-modified A-DROP scores showed the same superiority, which confirmed the robustness of the predictive ability of chest CT modification.

In a review of the literature, one prospective study showed that chest CT contributed more to the diagnostic accuracy of CAP than did chest radiograph imaging in an emergency department setting [20]. No previous studies had evaluated the incremental predictability of chest CT. Our results may therefore provide physicians with new insights into an effective strategy for managing CAP. 


\section{TABLE 1 Baseline patient characteristics}

\begin{tabular}{|c|c|c|c|}
\hline Characteristics & Eligible patients & Registry population & p-value \\
\hline Subjects $n$ & 265 & 791 & \\
\hline Age years & $79.0(69.0-85.0)$ & $75.0(62.3-83.0)$ & $<0.001$ \\
\hline Male & $171(64.5)$ & $446(56.4)$ & 0.024 \\
\hline Treated in outpatient clinic & $0(0.0)$ & $338(42.7)$ & \\
\hline BMI $\mathrm{kg} / \mathrm{m}^{2}$ & $19.9(17.3-22.9)$ & $20.9(18.1-23.7)$ & 0.017 \\
\hline Performance status & & & $<0.001$ \\
\hline 0 & $84(31.7)$ & $383(49.1)$ & \\
\hline 1 & $53(20.0)$ & $165(21.2)$ & \\
\hline 2 & $37(14.0)$ & $82(10.5)$ & \\
\hline 3 & $43(16.2)$ & $75(9.6)$ & \\
\hline 4 & $48(18.1)$ & $75(9.6)$ & \\
\hline \multicolumn{4}{|l|}{ Comorbidity } \\
\hline Diabetes & 54 (20.3) & 150 (19.2) & 0.731 \\
\hline Chronic heart failure & $42(15.8)$ & 73 (9.3) & 0.005 \\
\hline Cerebrovascular disease & $66(25.0)$ & $124(15.8)$ & 0.001 \\
\hline Chronic kidney disease & $28(10.6)$ & $49(6.3)$ & 0.01 \\
\hline Dementia & $37(14.0)$ & $68(8.7)$ & 0.019 \\
\hline Malignancy & 59 (22.3) & $136(17.4)$ & 0.122 \\
\hline Chronic respiratory disease & 75 (28.3) & $243(30.7)$ & 0.248 \\
\hline Autoimmune disease & $20(7.5)$ & $48(6.1)$ & 0.506 \\
\hline CURB-65 & & & $<0.001$ \\
\hline 0 & 6 (2.3) & $23(2.9)$ & \\
\hline 1 & 31 (11.7) & $169(21.4)$ & \\
\hline 2 & 79 (29.8) & $274(34.6)$ & \\
\hline 3 & 95 (35.8) & $227(28.7)$ & \\
\hline 4 & $46(17.4)$ & 83 (10.5) & \\
\hline 5 & $8(3.0)$ & $15(1.9)$ & \\
\hline Chest radiograph & $265(100.0)$ & $791(100.0)$ & \\
\hline Chest CT scan & $265(100.0)$ & $527(67.7)$ & \\
\hline \multicolumn{4}{|l|}{ Sputum culture } \\
\hline Culture positive & 252 (95.0) & $737(93.1)$ & 0.268 \\
\hline CAP drug-susceptible pathogens & 220 (87.3) & $663(90.0)$ & 0.093 \\
\hline CAP drug-resistant pathogens & $32(12.7)$ & $74(10.0)$ & \\
\hline Virus PCR & 197 (74.3) & $552(70.0)$ & 0.987 \\
\hline Influenza virus & $8(4.1)$ & $23(4.1)$ & \\
\hline Parainfluenza virus & $10(3.8)$ & $22(2.8)$ & \\
\hline Human metapneumovirus & $6(3.0)$ & 18 (3.3) & \\
\hline Respiratory syncytial virus & $9(4.6)$ & $20(3.6)$ & \\
\hline Rhinovirus & $15(7.6)$ & 59 (10.7) & \\
\hline Died within $\mathbf{3 0}$ days after admission & $33(12.5)$ & $46(5.8)$ & 0.058 \\
\hline
\end{tabular}

Data are presented as median (interquartile range) or $\mathrm{n}(\%)$, unless otherwise stated. BMI: body mass index; CURB-65: confusion, urea $>7 \mathrm{mmol} \cdot \mathrm{L}^{-1}$, respiratory rate $\geqslant 30$ breaths $\mathrm{min}^{-1}$, blood pressure $<90 \mathrm{mmHg}$ (systolic) $\leqslant 60 \mathrm{mmHg}$ (diastolic), age $\geqslant 65$ years; CT: computed tomography; CAP: community-acquired pneumonia.

In previous studies, chest radiography was evaluated by radiologists [13-15]. In contrast, chest image evaluation by clinicians in this study was akin to that in a practical clinical setting, such as an emergency room. Although we have not excluded the prognostic predictability of chest radiographs per se, chest radiography-modified CURB-65 and A-DROP scores did not show superiority, in terms of the AUROC, for predicting 30-day mortality. The reproducibility of chest radiograph observations was also inadequate. Chest radiographs tended to under-detect pleural effusion, particularly in patients with a low ECOG performance status, history of chronic heart failure, and chest CT-determined infiltration affecting $\geqslant 4$ lobes. In contrast, chest CT observations were very reproducible. In particular, the presence of pleural effusion showed almost perfect inter-rater agreement. Assessment of multilobar involvement showed some inter-rater disagreement in terms of the recognition of old shadows, subtle infiltrates, ground-glass opacities and granular shadows, but in terms of involvement of $\geqslant 4$ lobes, there was substantial inter-rater agreement. These findings might be advantageous for physicians who must evaluate them in a tertiary care hospital. 


\begin{tabular}{|c|c|c|c|c|c|}
\hline & & $\begin{array}{c}\text { Patients }^{\#} \\
\text { n (\%) }\end{array}$ & Score & Mortality rate $\%$ & p-value \\
\hline \multirow[t]{2}{*}{ Infiltrate on chest CT } & $<4$ lobes & $193(82.1)$ & 0 & 3.6 & \multirow{2}{*}{$<0.01$} \\
\hline & $\geqslant 4$ lobes & 42 (17.9) & 1 & 52.3 & \\
\hline \multirow[t]{2}{*}{ Pleural effusion on chest $\mathrm{CT}$} & Negative & $171(72.8)$ & 0 & 2.9 & \multirow{2}{*}{$<0.01$} \\
\hline & Positive & $64(27.2)$ & 1 & 37.5 & \\
\hline \multirow[t]{2}{*}{ Multilobar infiltration on chest radiograph } & Negative & $149(63.4)$ & 0 & 10.1 & \multirow{2}{*}{0.22} \\
\hline & Positive & $86(36.6)$ & 1 & 16.3 & \\
\hline \multirow[t]{2}{*}{ Pleural effusion on chest radiograph } & Negative & $228(97.0)$ & 0 & 11.8 & \multirow{2}{*}{0.21} \\
\hline & Positive & $7(3.0)$ & 1 & 28.6 & \\
\hline
\end{tabular}
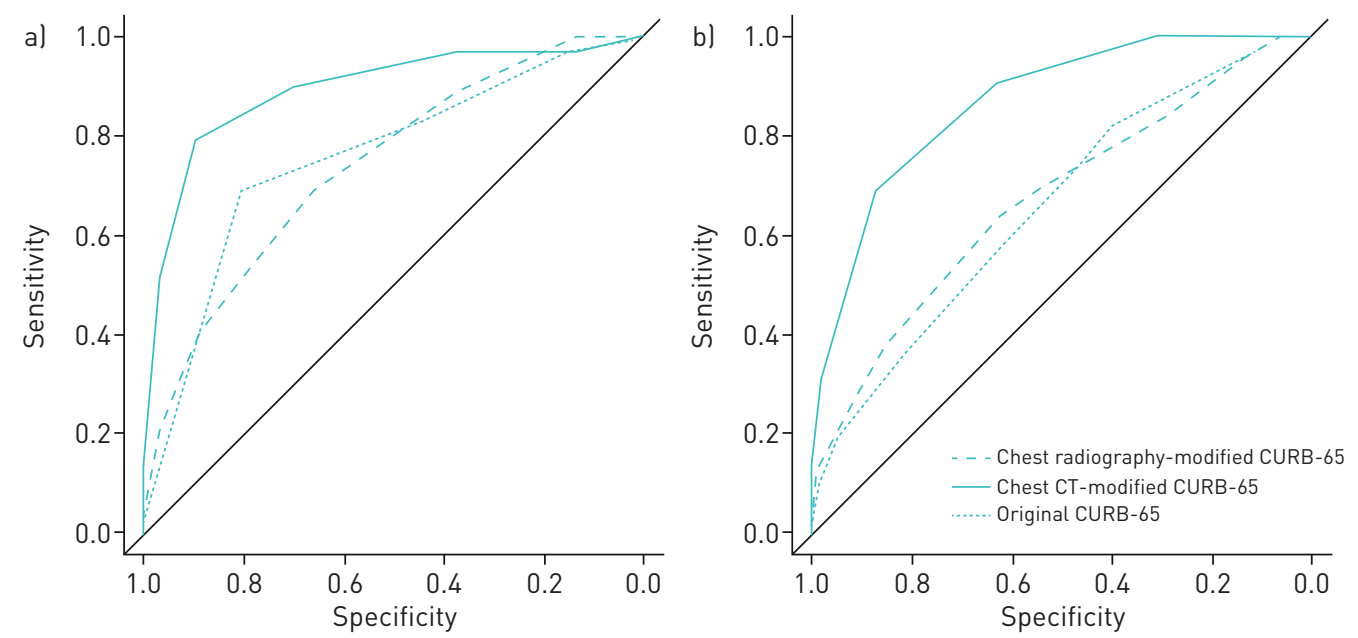

FIGURE 2 Area under the receiver operating characteristic curve for prediction of 30-day mortality using the original, chest radiography-modified, and chest computed tomography-modified a) A-DROP and b) CURB-65 (confusion, urea $>7 \mathrm{mmol} \cdot \mathrm{L}^{-1}$, respiratory rate $\geqslant 30$ breaths $\cdot \mathrm{min}^{-1}$, blood pressure $<90 \mathrm{mmHg}$ (systolic) $\leqslant 60 \mathrm{mmHg}$ (diastolic), age $\geqslant 65$ years scores.

\section{TABLE 3 AUROC for 30-day mortality for the various prediction scores}

\begin{tabular}{|c|c|c|c|c|c|c|}
\hline Severity score & $\begin{array}{c}\text { Optimal } \\
\text { cut-off score }\end{array}$ & AUROC & Difference $(95 \% \mathrm{CI})^{\#}$ & $\begin{array}{c}\text { Positive-predictive } \\
\text { value } \%\end{array}$ & $\begin{array}{c}\text { Negative-predictive } \\
\text { value } \%\end{array}$ & p-value* \\
\hline \multicolumn{7}{|l|}{ CURB-65 } \\
\hline CXR-modified CURB-65 & 3 & 0.664 & $0.006(-0.040$ to 0.056$)$ & 26.2 & 87.4 & 0.795 \\
\hline Chest CT-modified CURB-65 & 4 & 0.866 & 0.207 (0.136 to 0.283$)$ & 80.8 & 78.6 & $<0.001$ \\
\hline \multicolumn{7}{|l|}{ A-DROP } \\
\hline Chest CT-modified A-DROP & 4 & 0.891 & $0.134(0.081$ to 0.194$)$ & 89.5 & 79.8 & $<0.001$ \\
\hline \multicolumn{7}{|c|}{$\begin{array}{l}\text { AUROC: area under the receiver operator characteristic curve; CURB-65: confusion, urea }>7 \mathrm{mmol} \cdot \mathrm{L}^{-1} \text {, respiratory rate } \geqslant 30 \text { breaths.min }{ }^{-1} \text {, } \\
\text { blood pressure }<90 \mathrm{mmHg} \text { (systolic) } \leqslant 60 \mathrm{mmHg} \text { (diastolic), age } \geqslant 65 \text { years; CT: computed tomography; CXR: chest radiograph; NA: not } \\
\text { applicable. " : difference of AUROC from the original score. *: compared with each original score (CURB-65 or A-DROP) of AUC. p<0.001, for } \\
\text { comparison between chest CT-modified and CXR-modified CURB- } 65 \text { and A-DROP values. }\end{array}$} \\
\hline
\end{tabular}


As for the predictive value of chest CT-modified CURB-65 and A-DROP scores, the high sensitivity (both $89.7 \%)$ and negative-predictive value $(97.8 \%$ and $97.9 \%$, respectively) of these systems for scores $\geqslant 3$ might also be useful for deciding site of care. In contrast, a previous study reported the sensitivity and specificity of a CURB-65 score $\geqslant 2$ as $92.8 \%$ and $49.2 \%$, respectively [8]. Another study suggested that the underestimation of the CURB-65 in older adults may be due to the inappropriate weight given to age [21]. The current study investigated one of the most elderly societies in the world [22]. In Japan, the incidence of CAP among people aged $\geqslant 65$ years was 42.3 per 1000 per year; meanwhile, the CAP incidence was 28.4 per 1000 per year in the USA [23], 14 per 1000 per year in Spain [24], and 8 per 1000 per year in the UK [25]. Herein, the findings that the AUROC for the A-DROP were numerically higher than those for the CURB-65, and that the PSI did not show higher AUROC than the original CURB-65 scores, might be explained by the age characteristics of our sample. Although the updated US guidelines in 2019 recommend using PSI based on several prospective studies from 2000 to 2005 [9], the PSI might not be a good fit for rapidly aging societies like Japan.

As facility-limited usage, cost and radiation exposure are often considered reasons to avoid chest CT in a clinical setting, the true value of chest CT might be underestimated due to a lack of research-based evidence. The current study not only provided additional academic insights to improve our knowledge of chest CT, but also demonstrated a practical application. In the sub-analysis comparing the characteristics of patients with or without radiological discrepancy, poor physical status, a history of chronic heart failure and multilobar infiltration were identified as factors that increase the difficulty of chest radiograph evaluation. Another reason for the discrepancy, which could not be addressed in the current study, was the presence of infiltration in the lower regions of the lung. This might complicate the detection of pleural effusion on the chest radiographs, whereas there would be no such difficulty with CT. As the efficiency of chest CT usage has improved, cost-effectiveness can also be improved by using chest CT for difficult patients with chronic thoracic disease or for patients who cannot maintain their posture for chest radiographs or cannot be assessed accurately due to comorbid cardiac disease [22-25]. Although we did not assess the prognostic value of other comorbidities in the lung, especially emphysema and fibrosis, those lung conditions might be reflected in the respiratory score in CURB-65, A-DROP and PSI. Further studies are needed to improve the prognostic ability of CT alone.

This study had several limitations. First, it used a registry of prospectively enrolled CAP patients, but analysed a limited number of hospitalised patients, who were relatively older and sicker, due to lack of manpower to evaluate the CT image data of all 792 participants in order to include outpatients. Therefore, the results of this study might not be generalisable to all patients. Nevertheless, the current sub-analysis showed that specific patients with a low ECOG performance status or history of chronic heart failure might particularly benefit from undergoing chest CT for severity assessment and site of care decisions. Second, an external validation for chest CT-modified scoring was not tested. Thus, a population-based prospective study is required to verify our findings; however, this requires a large population and high costs, and the results may still not be applicable to real-world practice because chest CT for the detection of pneumonia is not routinely recommended for facility-limited equipment, higher cost and radiation exposure. Therefore, multiple studies and meta-analyses are needed to make recommendations regarding the use of chest CT in the practice of CAP.

\section{Conclusions}

In conclusion, chest CT-modified scores were shown to improve the prediction of 30-day mortality in patients with CAP. Further large-cohort, multinational research studies are needed to confirm our results.

Acknowledgements: We would like to thank Editage (www.editage.jp) for English language editing. We are also grateful to the staff of the Adult Pneumonia Study Group - Japan (APSG-J) study, in particular, the staff of the Depts of Pulmonary Medicine, Infectious Diseases, Laboratory Medicine and General Internal Medicine, and the Emergency and Trauma Center at Kameda Medical Center, Chiba, Japan.

Author Contributions: M. Nemoto, K. Nakashima, Y. Matsue, K. Yoshida, Y. Matsui, and M. Aoshima contributed to the study's conception and design. K. Nakashima and M. Aoshima provided administrative support. All authors were involved in provision of study materials or patients. K. Nakashima and M. Nemoto were involved in collection and assembly of data. All authors were involved in data analysis and interpretation. All authors were involved in manuscript writing. All authors read and approved the final version of the manuscript.

Conflict of interest: M. Nemoto has nothing to disclose. K. Nakashima has nothing to disclose. S. Noma has nothing to disclose. Y. Matsue has nothing to disclose. K. Yoshida has nothing to disclose. H. Matsui has nothing to disclose A. Shiraishi has nothing to disclose. T. Ishifuji has nothing to disclose. K. Morimoto reports grants and personal fees from Pfizer, and personal fees from MSD Pharma, outside the submitted work. K. Ariyoshi has nothing to disclose. M. Aoshima has nothing to disclose. 


\section{References}

1 GBD 2017 Causes of Death Collaborators. Global, regional, and national age-sex-specific mortality for 282 causes of death in 195 countries and territories, 1980-2017: a systematic analysis for the Global Burden of Disease Study 2017. Lancet 2018; 392: 1736-1788.

2 Mandell LA, Wunderink RG, Anzueto A, et al. Infectious Diseases Society of America/American Thoracic Society consensus guidelines on the management of community-acquired pneumonia in adults. Clin Infect Dis 2007; 44 Suppl. 2, S27-S72.

3 Woodhead M, Blasi F, Ewig S, et al. Guidelines for the management of adult lower respiratory tract infections. Clin Microbiol Infect 2011; 17: Suppl. 6, E1-E59.

4 National Institute for Health and Care Excellence. Pneumonia in adults: diagnosis and management. Clinical guideline [CG191]. www.nice.org.uk/guidance/cg191. Date last accessed: 6 December 2019: date last updated: September 2019.

5 Miyashita N, Matsushima T, Oka M, et al. The JRS guidelines for the management of community-acquired pneumonia in adults: an update and new recommendations. Intern Med 2006; 45: 419-428.

6 Shindo Y, Sato S, Maruyama E, et al. Comparison of severity scoring systems A-DROP and CURB-65 for community-acquired pneumonia. Respirology 2008; 13: 731-735.

7 Jones BE, Jones JP, Vines CG, et al. Validating hospital admission criteria for decision support in pneumonia BMC Pulm Med 2014; 14: 149.

8 Lim WS, van der Eerden MM, Laing R, et al. Defining community acquired pneumonia severity on presentation to hospital: An international derivation and validation study. Thorax 2003; 58: 377-382.

9 Metlay JP, Waterer GW, Long AC, et al. Diagnosis and treatment of adults with community-acquired pneumonia An official clinical practice guideline of the American Thoracic Society and Infectious Diseases Society of America. Am J Respir Crit Care Med 2019; 200: e45-e67.

10 Syrjala H, Broas M, Suramo I, et al. High-resolution computed tomography for the diagnosis of community-acquired pneumonia. Clin Infect Dis 1998; 27: 358-363.

11 Okada F, Ono A, Ando Y, et al. Thin-section CT findings in Pseudomonas aeruginosa pulmonary infection. Br J Radiol 2012; 85: 1533-1538.

12 Prina E, Ranzani OT, Torres A. Community-acquired pneumonia. Lancet 2015; 386: 1097-1108.

13 Charles PG, Wolfe R, Whitby M, et al. SMART-COP: a tool for predicting the need for intensive respiratory or vasopressor support in community-acquired pneumonia. Clin Infect Dis 2008; 47: 375-384.

14 Fine MJ, Smith MA, Carson CA, et al. Prognosis and outcomes of patients with community-acquired pneumonia. A meta-analysis. JAMA 1996; 275: 134-141.

15 Hasley $\mathrm{PB}$, Albaum MN, Li YH, et al. Do pulmonary radiographic findings at presentation predict mortality in patients with community-acquired pneumonia? Arch Intern Med 1996; 156: 2206-2212.

16 Morimoto K, Suzuki M, Ishifuji T, et al. The burden and etiology of community-onset pneumonia in the aging Japanese population: a multicentre prospective study. PLoS One 2015; 10: e0122247.

17 Hamaguchi S, Suzuki M, Sasaki K, et al. Six underlying health conditions strongly influence mortality based on pneumonia severity in an ageing population of Japan: a prospective cohort study. BMC Pulm Med 2018; $18: 88$.

18 Hornik K. R - The Comprehensive R Archive Network. Wiley Interdiscip Rev Comput Stat 2012; 4: 394-398.

19 Kanda Y. Investigation of the freely available easy-to-use software 'EZR' for medical statistics. Bone Marrow Transplant 2013; 48: 452-458.

20 Claessens YE, Debray MP, Tubach F, et al. Early chest computed tomography scan to assist diagnosis and guide treatment decision for suspected community-acquired pneumonia. Am J Respir Crit Care Med 2015; 192: 974-982.

21 Chen JH, Chang SS, Liu JJ, et al. Comparison of clinical characteristics and performance of pneumonia severity score and CURB-65 among younger adults, elderly and very old subjects. Thorax 2010; 65: 971-977.

22 Muramatsu N, Akiyama H. Japan: Super-aging society preparing for the future. Gerontologist 2011; 51: 425-432.

23 Jackson ML, Neuzil KM, Thompson WW, et al. The burden of community-acquired pneumonia in seniors: results of a population-based study. Clin Infect Dis 2004; 39: 1642-1650.

24 Vila-Corcoles A, Ochoa-Gondar O, Rodriguez-Blanco T, et al. Epidemiology of community-acquired pneumonia in older adults: a population-based study. Respir Med 2009; 103: 309-316.

25 Millett ER, Quint JK, Smeeth L, et al. Incidence of community-acquired lower respiratory tract infections and pneumonia among older adults in the United Kingdom: a population-based study. PLoS One 2013; 8: e75131. 